\title{
Protecting bottlenose dolphins from coastal construction
}

Professor Ann Weaver of Good-Natured Statistics Consulting, USA has studied dolphins ling free at sea for 20 years. Her numerous scientific discoveries about dolphin behaviour yield ample evidence of intelligence and of sophisticated social behaviour at sea. Drawing from many years of stophive studles in primates, she undertook an impressive 18-year ethological study of the social behaviour and impact support the need for management of coastal construction projects and surrounding areas to ensure local aquatic life has access to a safe place, away from noisy building works.
B ottlenose dolphins (Tursiops and sleek swimmers can be very playfu and can often be seen leaping out of only do they have incredible acrobatic skills, dolphins also express complex sets of behaviours, including the food, using various objects like toys behaviours, and even on sycasion teasing researchers studying them. as well as help humans. These graceful the water to follow nearby boats. Not practice of controlled conflict, sharing
Sadly, more and more dolphins also encroaching on their habitat. The accelerating pace of construction in coastal zones has turned these areas in on Earth. Noise pollution in pasticular is a serious issue for cetaceans: these animals depend on sound to navigate, communicate with other dolphins avoid predators, and search for food. This makes them highly vulnerable to noise. However, research looking at how coastal construction can affect dolphins is limited and often inadequate, even though it's urgently needed to protect these marine species.

\section{THE BRIDGE}

For many years, Weaver has been trying to cover this gap. The perfect research opportunity presented itself in the form of a bridge replacement project over on the west-central coast of Florida. The project involved removing the old bridge and building a new one, which included driving countless piles as bare moorings on the east side facing the Intra-Coastal Waterway and the west side facing the Gulf of Mexico. Inevitably the bridge removal and replacement large floating structures that would not be there otherwise.

Most bottlenose dolphins like the amiliarity of the same locations year after year, making them particularly vulnerable to long-term disruptions like the ones caused by construction
sites. The impact of these human have to deal with urban developments a narrow corridor frequently used by project involved increased noise levels, illumination, vessel traffic, and various

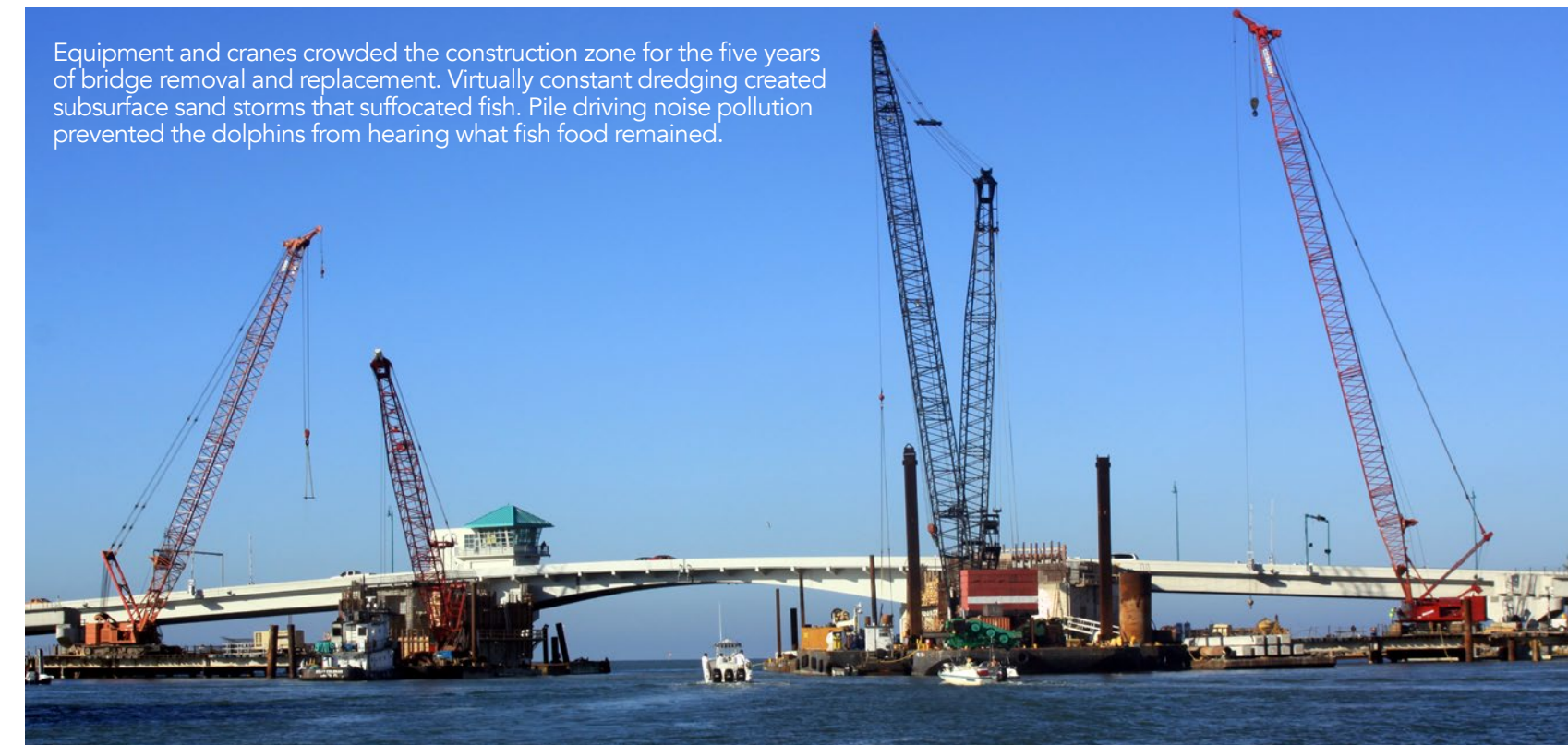

activities on cetacean behaviour is not easy to follow - as most of it happens "bridge construction project over John's Pass provided the perfect opportunity female sightings, but males continued to feel comfortable enough to visit the spotted twice as often as males, bute a 25\% drop during construction and a further $25 \%$ reduction after construction, to document freerect dolphin behaviour before, during, and dolphin behaviour before, during, and $\quad$ it became just as hard to spot a female
after bridge construction". Weaver drew
as it was to spot a male. Weaver upon 15 years of

way a dolphin mother and her calf communicate with whistles and other contact, and anything that interferes with maintaining contact jeopardises cal survival. Weaver's results suggested that dolphin mothers prefer quieter waters than males to "talk" to their offspring

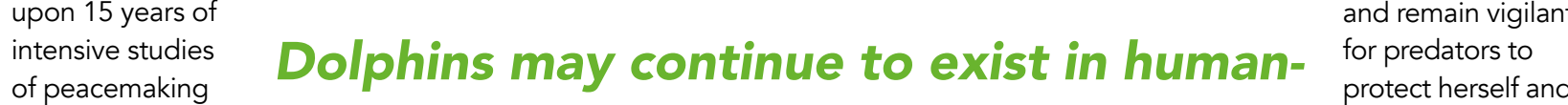
of peacemaking degraded habitats, but that does not hercalf.
and socill development in primates, with the necessary observational skills characterised this as a slow, steady leak this decline in females will do to mean that construction was neutral.

It's too early to understand what rather than an abrupt departure from the
area, implying that "old habits die hard".
Bottlenose dolphins reproduce slowly, Botlenose dolphins reproduce slowly,

\section{FEMALES VS MALES}

nitially, Weaver wanted to test whether males and females reacted the same way to the bridge replacement project. "Although a sex difference is predicted by bottlenose dolphin natural histor nothing was known about how sex differences might interact with long-term construction projects in key areas of dolphin habitat", says the behavioura researcher.

In a nearby project, bridge construction in Sarasota forced local dolphins to disperse, but numbers recovered once Pass, however it mas a mol. In John's Pass, however, th was a more complex story. The bridge replacement was
associated with a steady decline in
For Weaver, these results did not come as a surprise. Females with young noisy disturbances than males. In fact; area were raising at least one calf. The

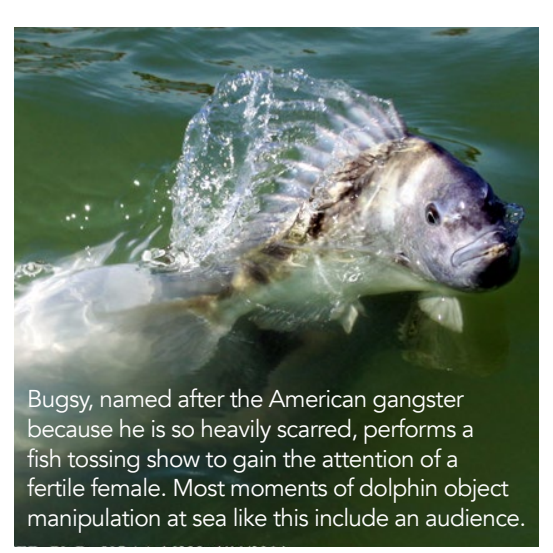
females observed abandoning the years to come Howet bo losing most of its females is never good for any population in the long term.

JUST A LITTLE LATER

After 11 years of sightings along John's Pass and the surrounding area, it turned out it wasn't just the ratio of females and males that was affected by this construction. Dolphins had to che feeding areas outside the construction zone. Sightings near the effects continued to be visible for five years after construction had ended. These "habitual creatures only chang what human impacts force them to change", explains Weaver. "When construction destroys their habitat 
elsewhere as long as suitable habitat is available to them

Weaver believes this change in eating such as pile driving and dredging So from the impact of a hammer hitting a pile causes waves around the piles, transmitting the noise through the water In fact, as the sound travels down the pile and into the water, it is noisier further from the pile than closer to it. Dredging creates underwater sand storms th suffocate fish. Not surprisingly, these devastating environmental impacts forced the dolphins to look for their next mea somewhere else.

In a domino effect, change in feeding patterns led to a series of other changes. Before cod to a 60 , spot as many as 60 dolphins foraging, Pass, sometimes for hours. Weaver documented over 9,000 sightings between 2006 and 2013. Across the year, these dolphins also showed minimigrations by moving out of the shallow intra-coastal waterways to spend the colder months in deeper waters offsho before returning during late winter and spring, and occupying their habituad waterways once again by summer.

However, it became increasingly harder to find dolphins during the first phase of construction. The number of these mammals visiting John's Pass simply dropped, and their routine migration completely disappeared. Dolphins construction phase, but they were stil wary and cautious. Intriguingly, John' Pass dolphins learnt to avoid the noise by moving their schedule of travelling and socialising to later in the day, coinciding with quieter periods whe construction activities were minimal. "They may continue to exist in humandegraded habitats, but that does not mean that construction was neutral" explains Weaver.

It may sound somewhat strange that dolphins changed their feeding habits but continued visiting the area for social purposes. According to Weaver it suggests that dolphins may change response to human disruption. On one

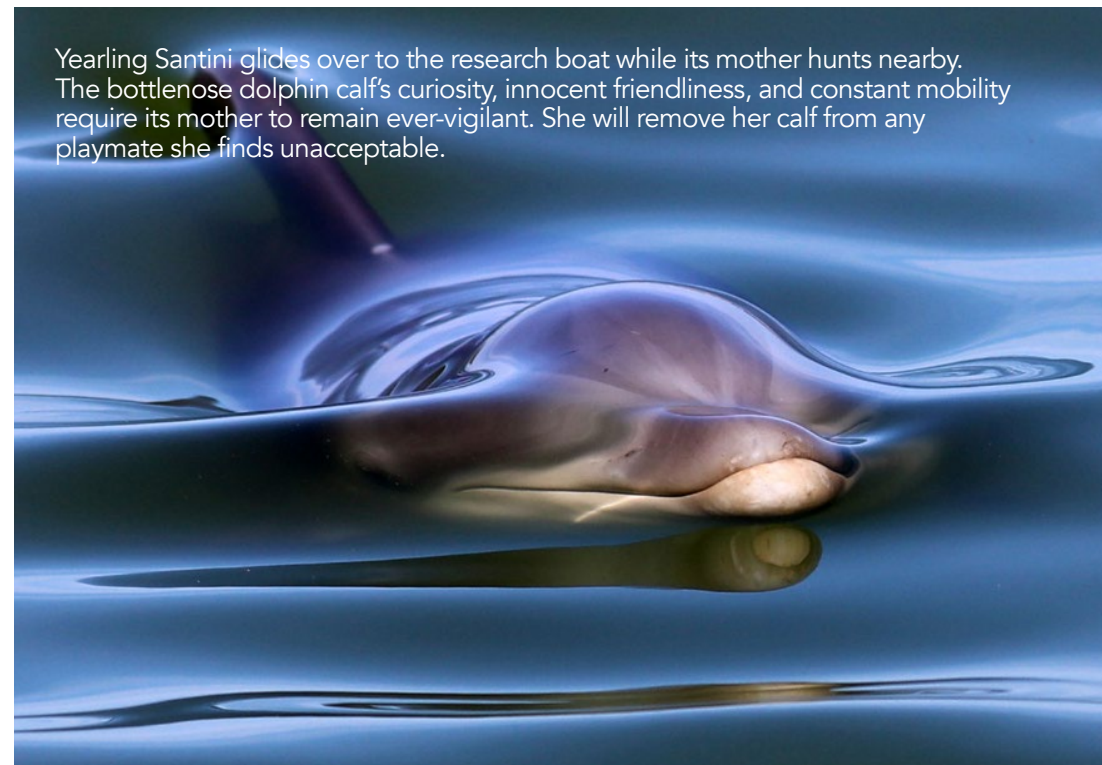

hand, dolphins were forced to move out of the construction zone to find food

chance to become familiar with because fish numbers were depleted. along John's Pass because there were still dolphins willing to stay in the area It turned out that feeding was more susceptible to changes in response to the bridge replacement, but since socia behaviour may not need a quiet place, and perhaps adapt in the second phase. Along with other researchers, Wearea also tresses that it should be required by law to assess how marine mammals and fish communities use the habitat before any coastal construction project can start.

These evaluations can then be used to identify and protect alternative habitats

This matter deserves more attention if we wish to maintain these dolphin populations.

to environmental impacts. Weaver's

$\begin{array}{ll}\text { to environmental impacts. Weaver's } & \text { nearby, giving the animals a safe haven } \\ \text { evidence is the first to suggest that human } & \text { away from noisy dredging, pile driving }\end{array}$ impacts may not affectall of a species' behaviours the same way an important warning to other researchers.

According to Weaver, this means that "the presence of socialising dolphins in construction zones can mislead observers For her, it is imperative that other behavioural adaptations are taken into account by researchers to assess the impact of coastal construction projects on coastal cetaceans.

RECOMMENDATIONS FOR FUTURE PROJECTS

Based on these observations, Weaver believes it's vital to monitor every develop a pout above ambient levels was not measured the current study but it clearly occurred", says Weaver. "Acoustic data are needed on construction-induced changes to the ambient acoustic environment to understand how such changes may

These suggestions are particularly relevant, as the US Department of Transportation's Federal Highway Administration has a list of over 4,000 bridges in Florida that need urgent repar work. This means it's highly likely that place in waters where dolphins abound. if we wish to meserves more attention "populations", concludes the rosin away from noisy dredging, pile driving, compromise vital ecological conditions". further construction projects will take
Detail

Bio

Animal behaviourist Ann Weaver has experience with over 200 species. She has academic "dual citizenships" in primatology (bonobos, capuchins, macaques) and bottlenose dolphins. She specialises in intensive ethological studies of individual animals recognised on sight. Ann works as a statistical consultant, and gublic interest in animals, nature, and science.

\section{Collaborators}

John Heidemann, Beth Brady, Brianna Seay Harvey, Kirsten Smail, Lesley Ferguson, Shauna Kebert, Casey Hamel, Amy Walters, Marie Dahlberg, Doris Eaton, and

Frans de Wa

\section{References}

- Weaver, A (2021). An ethology of adaptation: Dolphins stop feeding but continue socializing in construction-degraded habitat. Front. Mar. Sci.

-Weaver, A (2020). Why dolphins jump - A picture book of the acrobats of the sea. Treasure Island $F$ Jaasas Acade

Weaver, A (2017). Secrets behind the dolphin smile 978-1973500360.

- Weaver, A and Kuczaj, S (2016). Neither toy nor tool: Grass-wearing behavior among free-ranging bottlenose dolphins in western Florida. Int. J. Comp. Psychol. 29. https://doi.org/10.5070/P4291031885. - Weaver, A (2015). Sex differences in bottlenose dolphin sightings during a long-term bridge construction project. Animal Behavior and

Cognition, 2(1), 1-13.

- Weaver, A (2003). Conflict and reconciliation in captive bottlenose dolphins, Tursiops truncatus.

- Weaver, A and de Waal, F (2003). Mother-offspring relationship as a template in social development apella). J. Comp. Psychol. 117(1), 101-110.

\section{Research Objectives}

In the longest and most intensive study of its kind, Ann Weaver's scientific discoveries shed light on the need to

\section{Personal Response}

Drawing upon findings of your research, you've made recommendations for protecting bottlenos dolphins from coastal construction. What needs implemented?

II Responsible stewardship of the Earth is needed. from governments and construction companies to pay for data on every coastal construction project. The cost of securing sound scientific guidance before coastal construction starts is a fraction of lost tourism dollars when, without science, wildlife is gone when
construction ends, and so are the tourists. Science pays dividends.

\section{You've spent many years investigating dolphins and} dolphin behaviour. What first sparked your interest

II I love watching animals behave naturally in nature. If $I$ were as interested in human cultures as $I$ am in animal cultures, I would be an ambassador. Dolphins world that requires only the brains of a barnacle. As we crowd the coastlines at a frantic pace, I think, "What a ungracious way to say 'Thank You' to the only animals
who come out of nature freely to try and save people from drowning and from sharks".

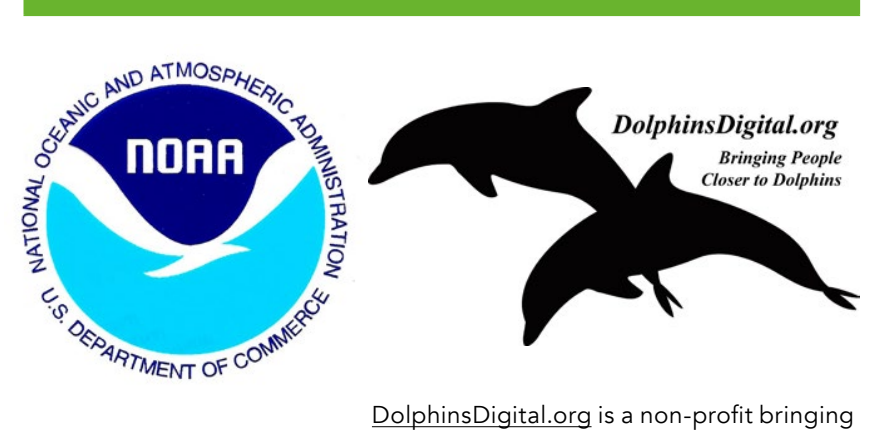

DolphinsDigital. org is a non-profit bringing
people closer to dolphins and finds ways to 